=

\section{Exploiting virtual globes for ecology and conservation in the Digital Earth era}

Because human activities are pushing the global ecosystem toward an irreversible state shift (Barnosky et al. 2012), there is a growing urgency for scientists to provide and effectively communicate solutions to large-scale environmental problems. The emergence of Digital Earth technologies - virtual representations of the Earth known as virtual globes (VGs), typified by Google Earth exemplify one of the tools that ecologists can use to address these crucial challenges (Goodchild et al. 2012).

We argue that ecologists, as compared with scientists in other disciplines, tend to underutilize VGs (WebFigure 1a). Here, we briefly review how ecologists have been using VGs and discuss current and potential applications of this technology in environmental research, policy, and management (WebFigure 2; WebTable 1). Although other VGs are available (eg WorldWind, GLOBE, Marble), we focus on the most used VG: Google Earth (Yu and Gong 2012) and its associated Google Street View (GSV).

VGs expand access to vast amounts of current and historical imagery beyond the contents of collections of individual researchers or institutions; within VGs, hundreds of thousands of aerial and satellite images are rapidly available to anyone with an internet-connected computer, smartphone, or other device. According to our literature search of publications in ecology and conservation, we found that, despite inherent limitations for studies that require highly accurate spatial resolution, use of imagery is Google Earth's primary application (75.4\%; WebFigure 2; WebTable 1). Additionally, GSV provides access to numerous high-resolution, onthe-ground, color images over large areas, allowing researchers and managers to remotely evaluate habitat at hitherto unavailable scales (Turner et al. 2003). Although rarely used, GSV imagery has proved to be helpful (1) to quantify fine-scale characteristics of species' habitats that would otherwise be difficult to study with other technologies (eg cliffs; Olea and Mateo-Tomás 2013), (2) to record species occurrence (Rousselet et al. 2013), and (3) to ground-truth data (Visser et al. 2014). Nonetheless, for ecological sampling, GSV is limited by several factors, including accuracy, lack of control by users over the timing of updates or when imagery was taken, and a bias toward highly visible flora and fauna close to roadsides or urban areas (WebTables 2 [link 1] and 3; Olea and Mateo-Tomás 2013). Yet the ongoing expansion of GSV over space - to unpaved roads and hiking trails, as well as to marine ecosystems (WebFigure 2; WebTable 2 [links 2-5]) - and over time (WebTable 2 [link 6]) shows promise for future applications in environmental science.

VGs can augment the processing capabilities beyond those supported by individual researchers or institutions, and without the need for supercomputers. For instance, a 30-mresolution world map of annual change in forest cover from 2000 to 2012 was made using Google Earth Engine (WebFigure 2; Hansen et al. 2013). This planetary-scale, geospatial analysis platform was crucial for processing the 650000 LANDSAT scenes used to create the map (WebTable 2 [link 7]). Although we found that Google Earth Engine is only rarely used in ecological research (1.7\%; WebFigure 2; WebTable 1), this technology allows researchers to generate key information for environmental policy makers and natural resource managers (WebTable 2 [link 8]).

Given that satellite imagery is often vital for analyses yet prohibitively expensive to obtain, unfettered access to VG technologies particularly for developing countries, which often have limited resources to counteract environmental crises - can help to promote ecological research and management $(17.7 \%$ of the reviewed literature explicitly highlighted this issue; WebTable 1).

Scientists and managers can easily visualize and share environmental information stored in Google Earth KML (Keyhole Markup Language) files, even while in the field. For example, use of KML files to describe survey locations and sampling points can improve experimental repeatability and quality in ecology (Shapiro and Baldi 2012). Indeed, scientific journals currently offer platforms that allow readers to access, explore, and share published geographic data using KML files (eg Elsevier's Interactive Map Viewer; WebTable 2 [link 9]).

VGs enable effective communication and engagement between researchers, managers, policy makers, and the public. Visualization is a "powerful means to communicate" (Nature Editorial 2006; McInerny et al. 2014), and freely available VG technologies offer opportunities to convey important messages or research outcomes, as well as to engage the public in environmental issues. Indeed, in many ecological subdisciplines, communicating science can be more effective through a free, user-friendly VG that resembles the Earth itself (WebFigure 2; WebTable 2 [link 10]; Goodchild et al. 2012). For instance, Google Earth is increasingly used by ecologists to communicate research results (eg through TED talks; WebTable 2 [link 11]) and by politicians to discuss environmental issues (WebTable 2 [links 12 and 13]). Google Earth also offers opportunities to engage a growing citizen-science community (WebTable 2 [link 14]; Dickinson et al. 2012). Crowdsourcing practices are already being coupled with Google Earth imagery to conduct inexpensive research over extensive areas (WebFigure 2), eg to improve accuracy in global land-cover maps (Fritz et al. 2013) or in the taxonomic identification of the crowns of tropical trees (González-Orozco et al. 2010). 
VGs are rapidly changing the way that scientists, managers, policy makers, and members of the public interact with environmental data worldwide. Although not exempt from limitations (eg inconsistencies in spatiotemporal resolutions; WebTable 3), VGs not only provide a rich source from which to generate and share environmental information, from local to global scales, but also offer capabilities and applications that promote the science and communication of ecology. Increasing their use today may help equip the next generation of VG practitioners with improved tools to better face the challenges of global environmental change.

\section{Pedro P Olea ${ }^{1,2 *}$ and Patricia Mateo-Tomás ${ }^{2 \dagger}$}

${ }^{1}$ Departamento de Ecología, Universidad Autónoma de Madrid, Madrid, Spain *(pedrop.olea@uam.es); Instituto de Investigación en Recursos Cinegéticos (IREC), CSIC-UCLM-JCCM, Ciudad Real, Spain; ${ }^{\dagger}$ current address: Plaza de los Pueblos $91^{\circ} \mathrm{A}, 24900$, Riaño, León, Spain
Acknowledgements

We thank W Jetz for comments on an early draft.

Barnosky AD, Hadly EA, Bascompte J, et al. 2012. Approaching a state shift in Earth's biosphere. Nature 486: 52-58.

Dickinson JL, Shirk J, Bonter D, et al. 2012. The current state of citizen science as a tool for ecological research and public engagement. Front Ecol Environ 10: 291-97.

Fritz S, See L, You L, et al. 2013. The need for improved maps of global cropland. Eos Trans AGU 94: 31-32.

González-Orozco CE, Mulligan M, Trichon $\mathrm{V}$, and Jarvis A. 2010. Taxonomic identification of Amazonian tree crowns from aerial photography: taxonomic identification of Amazonian tree crowns. Appl Veg Sci 13: 510-19.

Goodchild MF, Guo H, Annoni A, et al. 2012. Next-generation Digital Earth. $P$ Natl Acad Sci USA 109: 11088-94.

Hansen MC, Potapov PV, Moore R, et al. 2013. High-resolution global maps of 21 st-century forest cover change. Science 342: 850-53.

McInerny GJ, Chen M, Freeman R, et al. 2014. Information visualisation for science and policy: engaging users and avoiding bias. Trends Ecol Evol 29: 148-57.

Nature Editorial. 2006. Think global. Nature 439: 763.
Olea PP and Mateo-Tomás P. 2013. Assessing species habitat using Google Street View: a case study of cliff-nesting vultures. PLoS ONE 8: e54582.

Rousselet J, Imbert C-E, Dekri A, et al. 2013. Assessing species distribution using Google Street View: a pilot study with the pine processionary moth. PLoS ONE 8: e74918.

Shapiro JT and Báldi A. 2012. Lost locations and the (ir)repeatability of ecological studies. Front Ecol Environ 10: 235-36.

Turner W, Spector S, Gardiner N, et al. 2003. Remote sensing for biodiversity science and conservation. Trends Ecol Evol 18: 306-14.

Visser V, Langdon B, Pauchard A, and Richardson DM. 2014. Unlocking the potential of Google Earth as a tool in invasion science. Biol Invasions 16: 513-34.

Yu L and Gong P. 2012. Google Earth as a virtual globe tool for Earth science applications at the global scale: progress and perspectives. Int J Remote Sens 33: 3966-86.

doi:10.1002/FEEOlealetter. 1

Supporting Information

Additional, web-only material may be found in the online version of this article at http://onlinelibrary. wiley.com/doi/10.1002/FEEOlea letter.1/suppinfo

\section{The United States National Vegetation Classification (USNVC) Your guide to inventorying vegetation communities}

The USNVC is the national framework for inventorying and studying all US vegetation. It provides a common language for the effective management and conservation of plant communities.

The full content of the USNVC is now available online! Explore the classification and discover how you can contribute to this vital, dynamic standard.

\section{usnvc.org}

